\title{
A Web-based Decision Support System with SMS-based Technology for Agricultural Information and Weather Forecasting
}

\author{
Jumoke Soyemi \\ Department of Computer Science, \\ The Federal Polytechnic, llaro. Nigeria
}

\author{
Adesi Adesola Bolaji \\ Department of Computer Science, \\ The Federal Polytechnic, llaro. Nigeria
}

\begin{abstract}
This study designed a framework to assist farmers increase their productivity by receiving weather information through decision support system. The system has been developed to keep track of weather information related to agriculture. With the growing population and demands to improve crop productivity; there is the need to make available sustainable resource practice that serves better both the communities and the nation. In satisfying this need, a web-based application which contains informative and insightful agricultural tutelages was developed to aid decision making in agro-processing, stimulate the farmer's climate information and provide useful information required to enhance crop productivity, especially in the rural areas. The application uses Short Message Service (SMS) Technology to disseminate weather forecasting to farmers according to their eWarning setup. Therefore, the Decision Support System with all the ready agricultural and weather information will be a huge advantage to farmers at large and is expected to impact positively on the present economy situation of the nation through increase in smallholder's productivity.
\end{abstract}

\section{Keywords}

Decision Support System, SMS Technology, Weather forecasting, Agricultural information, eWarning

\section{INTRODUCTION}

Several decisions made by management, in the modern day farming demand up-to-date and local information, especially for weather forecasting [1]. There existed varieties of opportunities for small-scale farmers to be converted into medium scale agricultural production. Amidst various approaches, availing decision support systems for enhancing access to agricultural information and sharing of agricultural insights including climate information is vital for actualizing improved crop production to smallholders. Hence, existing methods of information supply to small-scale farmers have been found to be non-effective in getting timely, comprehensible and actionable agricultural information and technologies. Such methods include television, extension agents and face to face communication.

Disseminating climate information and sharing agricultural information can be facilitated by application made available through Information Technology (IT). The existing methods of dissemination include radio, television, face to face, and electronic mail. Furthermore, web-based system has been developed and used to disseminate agricultural information. However, the use of web and mobile technologies has not been adequately utilized for this purpose [2].
SMS Technology can be adequately used for expert advice, agriculture assistance and veterinarian consulting for identification and management of livestock illnesses, crops and diseases or pest control, appropriate seed, pesticides, timely planting or harvesting techniques relating to weather predictions, financial transactions (consulting with lenders or financial loans), agriculture training, up-to-date price information for agricultural commodities, as well as contact details for interested buyers for rural farmers.

SMS is relatively cheap, robust and reliable technologies used to help small-scale farmers improve their agriculture business. The proper use of SMS between all agriculture stakeholders could improve the agriculture business which also improves the living standard of farmers. They are empowered through this means in making a timely decision if they have climate and weather status. If there is no proper communication between all stakeholders, it creates the misunderstanding and frustration which affect the quality of production and reduce the business, performance of work and cause delays. So to improve the farmers' financial condition and empower the farmers' needs, strong communication between farmers using the inexpensive mobile phone technology is the key.

This study, therefore, investigates decision support systems with SMS technology that small-scale farmers and agricultural extension workers at village levels can operate, understand and hence improves access to agricultural information, especially climate information and innovations and to support strategic farm decisions under a changing climate.

\section{LITERATURE REVIEW}

\subsection{Weather Forecasting in Agriculture}

Weather forecasting is defined as "prediction of the state of the atmosphere for a given location applying the principles of physics, supplemented by a variety of statistical and empirical techniques and by technology" [4]. Weather forecasts are important because they are designed to protect life and property, save crops and predict what to expect in the atmospheric environment.

Various authors have considered the evolution of the term and its usage and how this maps to improve agriculture generally. Science Daily, in a review of the term's usage, offered the definition of Weather forecasting as collecting as much data as possible about the current state of the atmosphere (particularly the temperature, humidity and wind) and using an understanding of atmospheric processes (through meteorology) to determine how the atmosphere evolves in the future [4]. All sources happen to agree with the fact that Weather Forecasting protects life and property, and improves farm productivity. 
The World Meteorological Organization, classified weather forecasting into different groups, identifying that, when the range is shorter, then the predictability becomes higher and more suitable for an agricultural forecast. The group classification ranges from Now-casting (between 0-2 hours), Very short-range forecast (up to 12 hours), Short range forecast (12 to 72 hours) and Long range forecast (12-30 days- 2 years) [9].

From another side, the elements of agricultural weather forecasts vary from place to place and from season to season, but they should refer to all weather elements, which affect farm planning and operations. The elements are Sky coverage by clouds, Precipitation, Temperature (maximum, minimum and dew point), Relative humidity, Wind Speed and direction, Extreme events (heat and cold waves fog, frost, hail, thunderstorms, wind squalls and gales, low pressure areas, different intensities of depressions, cyclones, tornados among others), Bright hours of sunshine, Solar radiation, Dew, Leaf wetness, Pan evaporation, Soil moisture stress conditions and supplementary irrigation for rain fed crops, Advice for irrigation timing and quantity in terms of pan evaporation, Specific information about the evolution of meteorological variables into the canopy layer in some specific cases, Microclimate inside crops in specific cases [9].

\subsection{Decision Support System}

Productivity of agriculture is dependent on a number of factors such as changing climate, harsh environmental conditions and changing commodity markets. Strategic decisions are required by farmers for seasonal response to such factors in order to maintain profitability. The decisions, including the crop variety to grow, the rate of sowing, fertilizer need and herbicide spraying with other agronomy practices, will contribute in a huge manner to the economical sustainability of individual farmer [3].

Hence, there is a need for the development of a Decision Support System assist farmers to make timely decisions. According to [8], Decision Support System is described as the collection of integrated software applications and hardware that form the backbone of an organization's decision making process. Also [6], defines decision support system as a system that assists management decision making by combining data, sophisticated analytical tools and user-friendly software into a single powerful system that can support structured, unstructured and semi structured decision making. However, [5] concluded that the main objective of DSS is to support and improve decision making.

DSS may be composed of four main components which are Database, Model Base, Knowledge Base and Graphics User Interfaces [5]. The functionality of data base is to store, retrieve and organize the raw data that will be used as information to make decisions in the knowledge engine component. The model base consists of the analytical capabilities of qualitative models. The functionality of the knowledge engine is designed to manage the problem-solving process, the problem recognition and the generation of a final solution. The user interface component is designed to facilitate users' interaction with the System [3].

Basically, there are three types of problems for decision making, namely structured, unstructured and semi-structured. Structured problems can be solved by standard solution techniques with clearly specified procedures to make a decision. Whereas the procedures of unstructured problems are unspecified in advance, and most of the decisions procedures are followed only once. In semi-structured problems, procedures for decision making can be specified but the optimal decision making cannot be verified [10].

In general, DSS is project-oriented and the functionality is always determined by system architecture. A typical DSS architecture should have an optimization toolbox, an on-line analytical processing toolbox, a data mining toolbox, a transaction processing system and a database as illustrated in Fig. 1.0. Users can generate problems to the DSS and obtain the analyzed answers from the user interface and a data mining toolbox can find the required data in large a database where data related to the problem are located.

\subsection{SMS Technology as eWarning}

Short Message Service is a store and forward way of transmitting short messages to and from mobiles [7]. The message from the source mobile is kept in a central short message center (SMS) which then forwards to the destination mobile. This implies that when the recipient mobile is not available; the short message is stored and can be sent later. The length of each short message cannot exceed 160 characters, while these characters can be text (alphanumeric) or binary Non-Text Short messages.

Unlike paging, SMS messages do not require the mobile phone to be active and within range, since they can be held for many days until the phone is active and within range. SMS messages are transmitted with the same cell or to anyone with roaming capability. They can also be sent from a computer to an ordinary mobile device such as the cellular phone and viceversa.

eWarning System is a communication strategy that provides people (in this case, smallholder farmers) early warning of all hazards, natural disasters, to create effective response actions to disasters before impact, thus dramatically reducing their effects on the lives of people, properties and in the case of farmers, crops and farm products. SMS Technology can be a great tool to give people the information they required when it comes to disaster preparedness because aside from being cheap, many smallholder farmers own at least one unit of cellular phone 


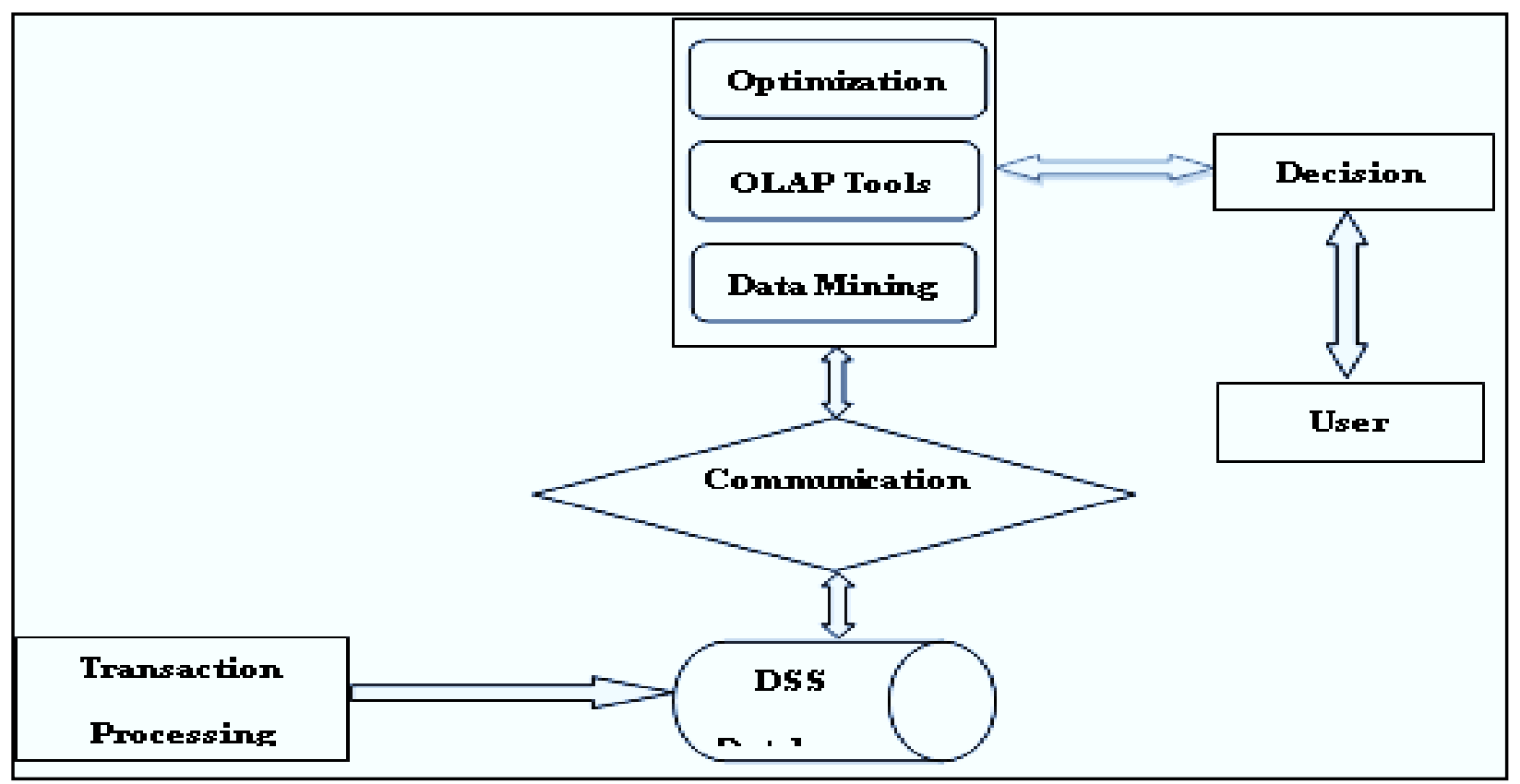

Fig:1 A typical structure of DSS

\section{SYSTEM DESIGN}

Systems are decomposed into smaller components where each component interrelates with other smaller system using defined interfaces with boundaries, specific purpose and constraints [2]. Figure 2 shows the decomposition of the web application to independent modules that interact with one another. Two main components of the web application are the weather forecast provider system and SMS gateway provider system. In this set up, users can interact with different platform on the web application to get desired information. For example, when administrators update the application, registered farmers and extension agents get such information through SMS. Similarly, when farmers and other users register themselves on the web platform, the administrators can view all registered farmers through the web interface on the admin page.

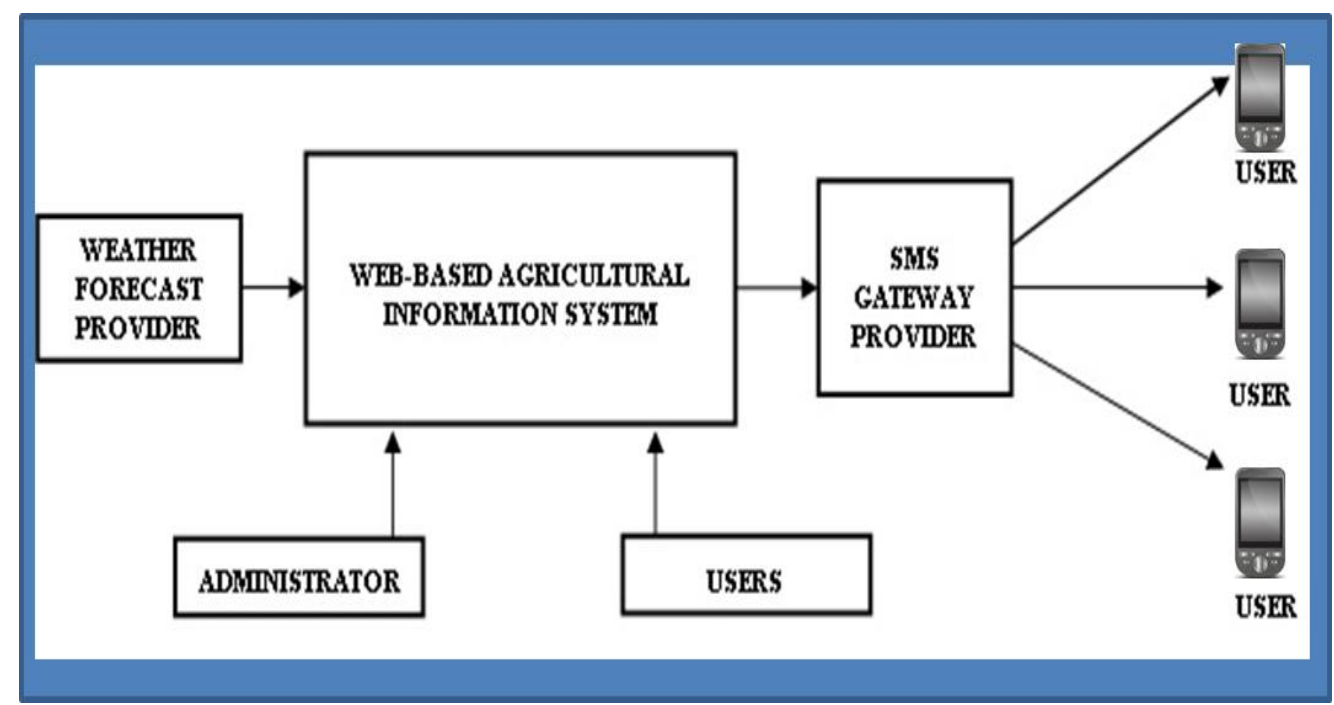

Fig 2: Proposed Web-based Agricultural Information System with SMS-based Technology Integration

Figure 3 shows unified model language (UML) diagram for modeling data of the application. The model was then used to implement a database using MySQL database management system (DBMS). The model design describes the interaction of the system with other sub-systems (environments). The system encompasses the database where the agricultural information resides, the eWarning System where the user set up a threshold to receiving weather information and the climate information system that extracts information directly from meteorological service.

Furthermore, the system is designed to supply information to its sub-systems. Hence, users get connected to the web application through an internet connection from a network provider using a computer system or mobile phone to assess the information on the web platform. 
The web application enables users to access agricultural information; sets up eWarning to get climate information and at the appropriate time to receive the climate information as a Short Message Service (SMS) from the application through SMS Gateway Provider.
Users can also get the current climate information on the web interface according to the user's input (city search). The web application is connected to open weather map; a meteorological service that provides daily weather forecast and other daily climate information

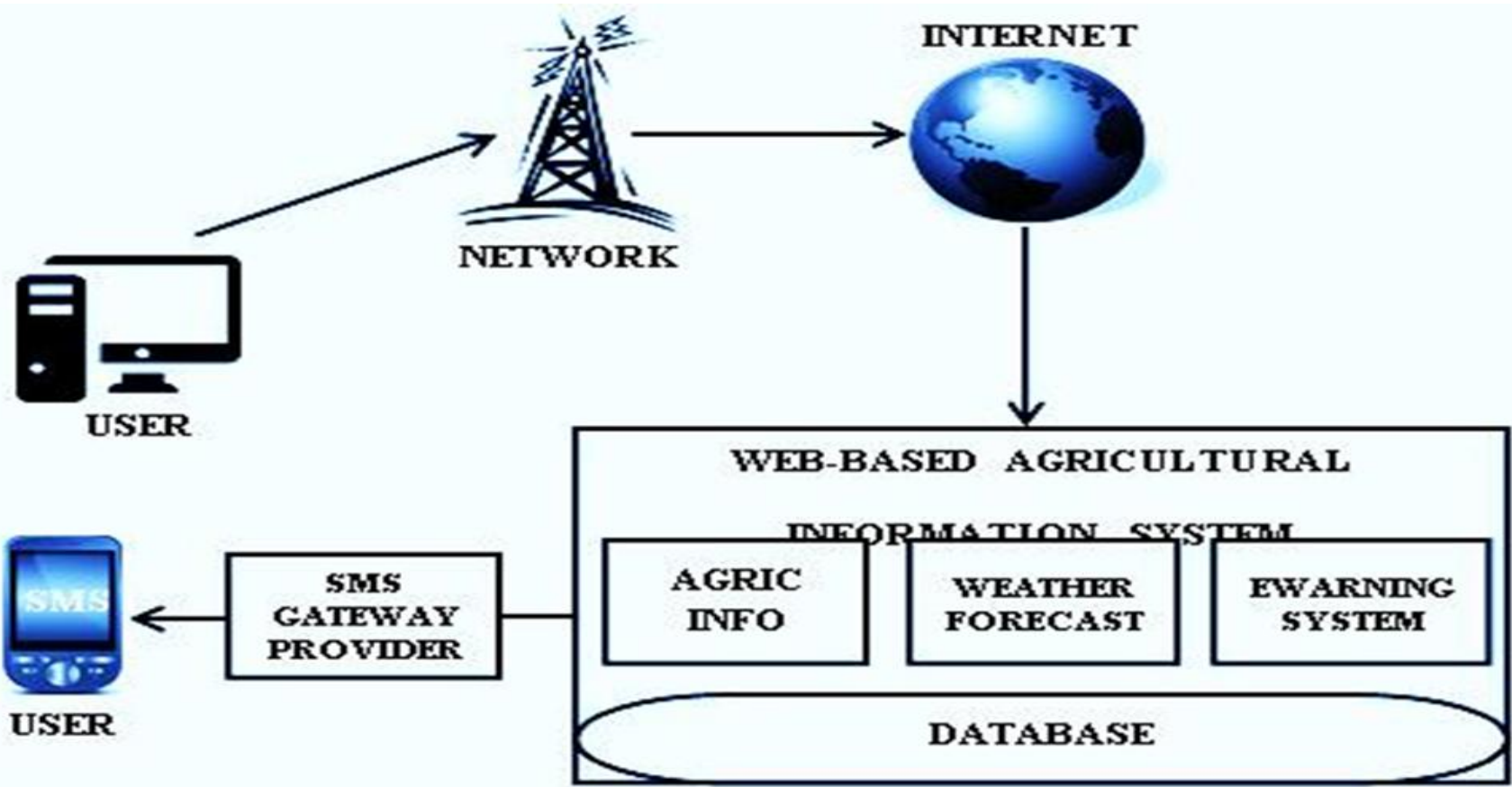

Figure 3: UML Diagram for Web-based Agricultural and Climate Information System

\section{SYSTEM IMPLEMENTATION}

The system designed was implemented to create the functions and operation of gathered requirements in detail, including screen layouts, process diagram and other documentations. The result of this stage describes the web application which is defined as a collection of modules and subsystems. For each requirement, there is a set of one or more design elements that are produced using the different models. All these design elements describe the desired software features, in detail, including functional hierarchy diagrams, screen layouts diagrams.

A web platform was suggested during the implementation phase because it provides online interaction among agricultural sectors.
In addition to the web platform, SMS-based technology was also used for implementation. The motivation of implementing SMS technology was driven by the needs of farmers in the rural area where the access to agricultural information for informed decision making was not encouraging.

Figure 4 shows the web interface with information about daily climate forecast for Ilaro areas, Nigeria of 2017 as updated by open weather map. The daily climate forecast showed the temperature, climate description, Humidity, Wind Speed, Sunrise and Sunset of the particular day. 


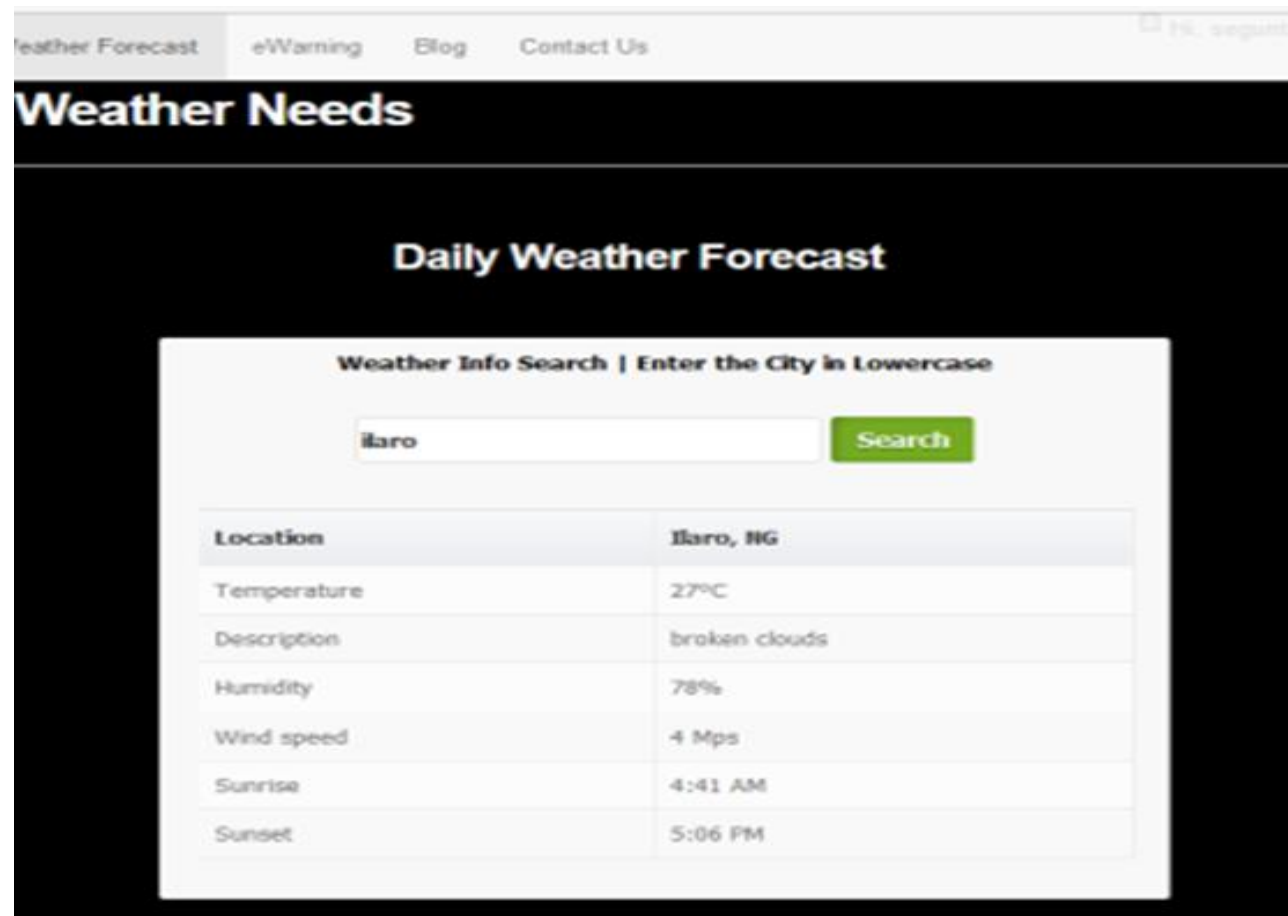

Figure 4: Web Interface for Daily Weather Forecast

Figure 5 shows part of web interface that displays platform for communicating alerts to registered users. Registered users who set up eWarning receives daily weather forecast when updated by open weather map through SMS by the systems administrator. The system allows farmers and other agricultural actors to register on the web interface using a computer system or mobile phone. Registered users can ask for daily climate forecast of the current season, and the system can find out if such information was updated and display forecast information to the user without human intervention.

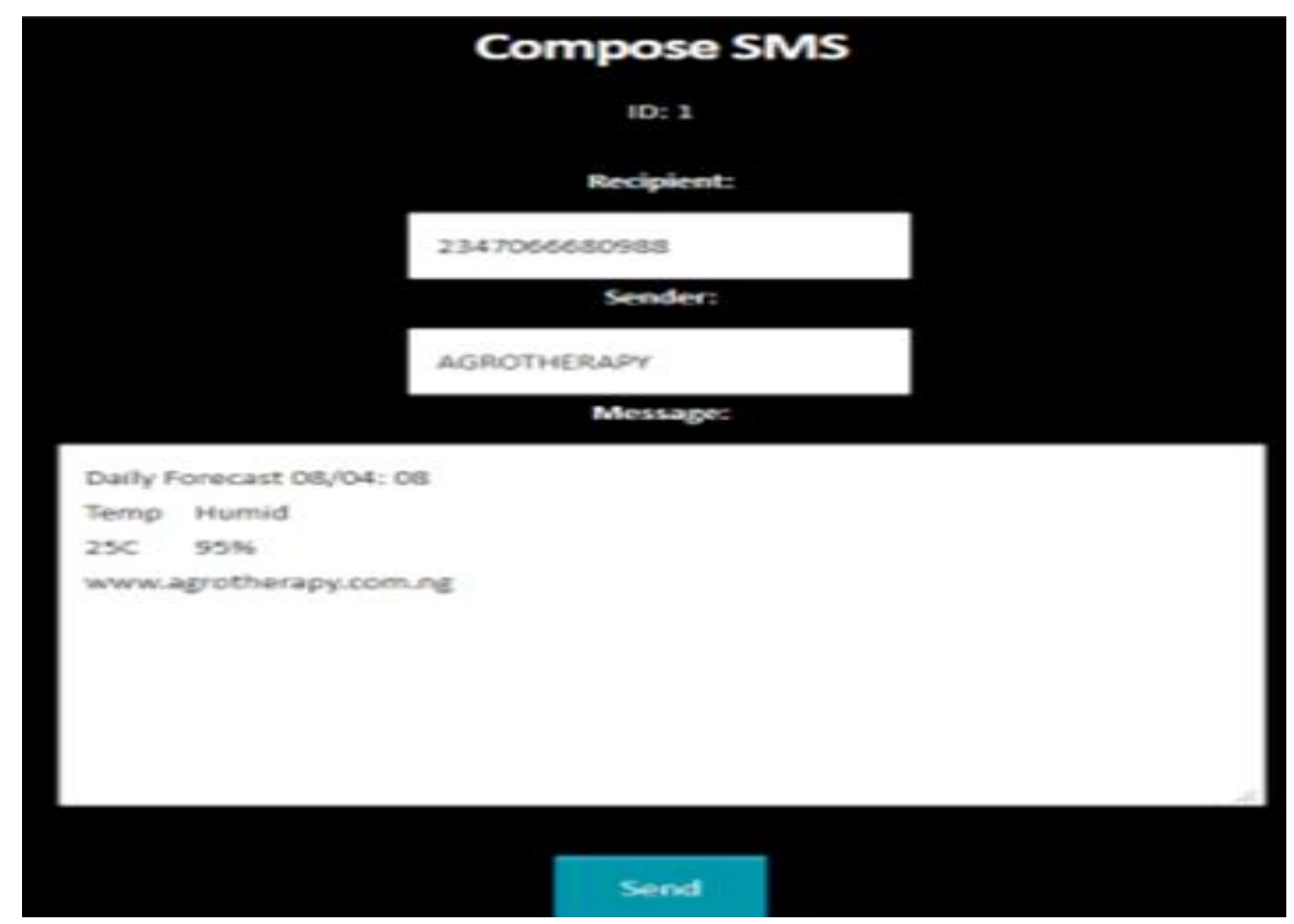

Figure 5: Web interface for communicating alerts to registered users

The climate information requested by the user through eWarning setup is sent manually by the systems administrator and this is one of the limitations of the web application; the ability to send climate information to users automatically. Hence, the system administrator gets the daily weather information as required by 
the user and manually types it into the web platform for communicating alerts and then send it to the contact provided by the registered user. Figure 6 shows the corresponding SMS as requested by the user on the web interface. The SMS is delivered directly to the registered user phone upon the action carried out by the system administrator who sent the climate information based on the user's request.
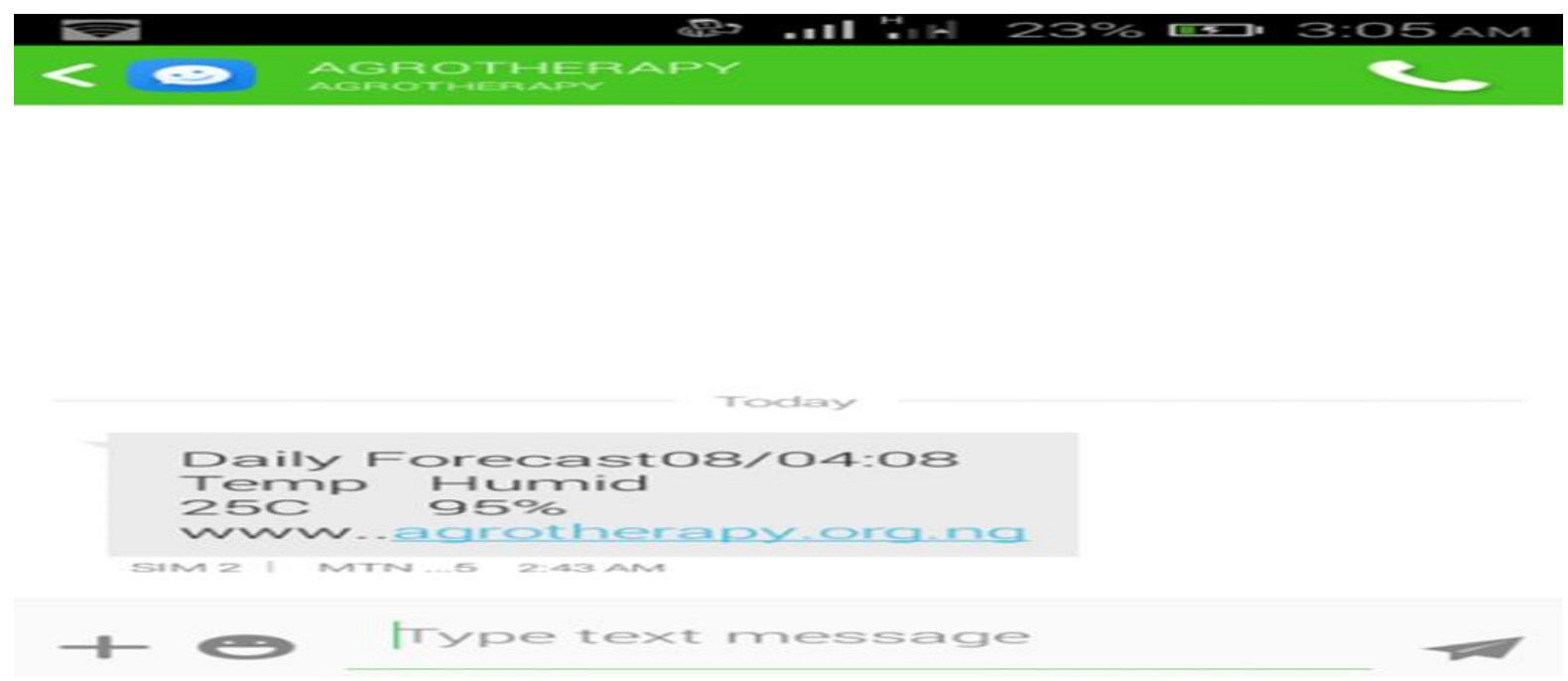

Figure 6: Corresponding SMS for Climate Information sent from Agrotherapy

\section{CONCLUSION}

The design and development of this application were purely based on RDMS, so that the large volume of queries could be handled easily. The Decision Support System developed contains informative and insightful agricultural tutelages to enhance farm operations and daily weather forecast to make reasonable decisions on plants, crops, soil and others. The contribution of agriculture to the development of any nation cannot be over-emphasized. The present situation of the nation will require governments to look beyond oil boom and revisit the agricultural sector with all that is needed to be put in place to take the full advantage of this system. The future scope of this research work can be extended to include getting local commodity prices on their mobile phone so as to make an informed decision and improve the system to the level where individual farmers can query the system through short codes.

\section{RECOMMENDATIONS}

The language of the system should be converted to local languages in order to be more useful for farmers in the rural areas. Further development of the economic model is required in order to address more complicated resource management patterns effectively. It is recommended that, more information relating to agriculture, particularly information coming from the agricultural sector of the government should be included into the system database. Also, information transferred to farmers can be increased substantially by providing email, news groups, messenger services, online chatting, and discussion groups. Further work should consider voice support in local languages for illiterate or semi-literate farmers.

\section{REFERENCES}

[1] IJ. Allan, "Agricultural Information and Decision Support by SMS,” EFITA 2003 Conference. pp.286-292, 2003.

[2] JC. Ayubu, RS. Mlozil, M. Henry, DT. Siza and C. Respickius, "A Decision Support System for Enhancing Crop Productivity of Smallholder Farmers in Semi-Arid Agriculture" International Journal of Information and
Communication Technology Research, col. 3, pp. 238-248, 2013.

[3] T. Pornchai, and A. Leisa, "Decision Support System Data for Farmer Decision Making", In Proceedings of Asian Federation for Information Technology in Agriculture, pp. 472-486, 2014.

[4] Science Daily, "Weather Forecasting”. Retrieved April 7, 2017

from:

https://www.sciencedaily.com/terms/weather_forecasting.ht $\mathrm{m}$. ScienceDaily features breaking news about the latest discoveries in science, health, the environment, technology, and more -- from major news services and leading universities, scientific journals, and research organizations.

[5] E. Turban, JE. Aronson, J.E.,and Liang, T.P. (2005), Decision Support Systems and Intelligent Systems(7th ed.). New Jersey, USA, Pearson Prentice Hall.

[6] R. Vansh, and M. Supriya, "Decision Support System, Its Components, Model and Types of Managerial Decision", International Journal of Innovative Research \& Studies, 2(12):413-418, 2013.

[7] KK. Veena, and VM. Thakare, "Short Message Service using SMS Gateway", (IJCSE) International Journal on Computer Science and Engineering, vol.2, pp. 1487-1491, 2010.

[8] ML. Waghmode, and PP. Jamsandekar, "Decision Support Systems and its Applications in different Sectors: Literature Review", International Journal of Advance Research in Computer Science and Management Studies, vol. 2, pp.110-115, 2014.

[9] WMO, "Guide to Agricultural Meteorological Practices (GAMP)", World Meteorological Organization (WMO), pp.134, 2010.

[10] HT. Zhang, Q. Yang, CS. Lai, and LL. Lai, "New trends for decision support systems". Paper presented at the IEEE International Conference on Systems, Man, and Cybernetic, 2012. 